\title{
Challenging Lorentz noninvariant neutrino oscillations without neutrino masses
}

\author{
V. Barger ${ }^{1}$, D. Marfatia ${ }^{2}$ and K. Whisnant ${ }^{3}$ \\ ${ }^{1}$ Department of Physics, University of Wisconsin, Madison, WI 53706 \\ ${ }^{2}$ Department of Physics and Astronomy, University of Kansas, Lawrence, KS 66045 \\ ${ }^{3}$ Department of Physics, Iowa State University, Ames, IA 50011
}

\begin{abstract}
We show that the combined data from solar, long-baseline and reactor neutrino experiments can exclude the generalized bicycle model of Lorentz noninvariant direction-dependent and/or direction-independent oscillations of massless neutrinos. This model has five parameters, which is more than is needed in standard oscillation phenomenology with neutrino masses. Solar data alone are sufficient to exclude the pure direction-dependent case. The combination of solar and long-baseline data rules out the pure direction-independent case. With the addition of KamLAND data, a mixture of direction-dependent and direction-independent terms in the effective Hamiltonian is also excluded.
\end{abstract}




\section{Introduction}

Neutrino oscillations are now a well-established phenomenon [1]. Data from solar, atmospheric, reactor and accelerator experiments may be explained by the now standard scenario with three active, massive neutrinos, with the possible exception of the LSND experiment [2]. Recently it has been suggested that Lorentz-invariance and $C P T$ violating interactions originating at the Planck scale can also lead to neutrino oscillations, with or without neutrino mass [3, 4, 5, 6, 7]. These interactions can be nonisotropic, which could lead to different oscillation parameters for neutrinos propagating in different directions.

The effective hamiltonian that describes the evolution of massless neutrinos in vacuum in the presence of Lorentz-invariance violating interactions may be written as [5]

$$
\left(h_{e f f}\right)_{i j}=E \delta_{i j}+\frac{1}{E}\left[\left(a_{L}\right)^{\mu} p_{\mu}-\left(c_{L}\right)^{\mu \nu} p_{\mu} p_{\nu}\right]_{i j},
$$

where $p_{\mu}=(E,-E \hat{p})$ is the neutrino four-momentum, $\hat{p}$ the neutrino direction, and $i, j$ are flavor indices. The coefficients $a_{L}$ have dimensions of energy and the $c_{L}$ are dimensionless. The Kronecker delta term on the right-hand side of Eq. (1) may be ignored since oscillations are insensitive to terms in $h_{\text {eff }}$ proportional to the identity. For antineutrinos, $a_{L} \rightarrow-a_{L}$.

Direction dependence of the neutrino evolution enters via the space components of $a_{L}$ and $c_{L}$. The coefficients may be specified in a celestial equatorial frame $(T, X, Y, Z)$, which has $Z$ axis along the Earth's rotation axis and $X$ axis towards the vernal equinox. The two-parameter bicycle model [5] can be defined as follows: $c_{L}$ is isotropic, with only one nonzero element $\left(c_{L}\right)_{e e}^{T T} \equiv 2 c$, and $\left(a_{L}\right)_{e \mu}^{\mu}=\left(a_{L}\right)_{e \tau}^{\mu}=(0, a \hat{Z} / \sqrt{2})$ are the only nonzero $a_{L}$. We have generalized the model by letting $\left(a_{L}\right)_{e \mu}^{\mu}=\left(a_{L}\right)_{e \tau}^{\mu}=(0, a \hat{n} / \sqrt{2})$, where $\hat{n}$ is the preferred direction for the $a_{L}$ interaction. This increases the number of parameters in the model to four, which is equal to the number required in the usual massive neutrino description of oscillations (two mass-squared differences and two mixing angles) [1]. We also consider a five-parameter model which has a linear combination of direction-dependent and direction-independent $a_{L}$.

In this letter we examine the phenomenology of this direction dependence in the generalized bicycle model with massless neutrinos. We find that the pure direction-dependent bicycle model is ruled out by solar neutrino data alone, while a combination of solar and long-baseline neutrino data excludes the pure direction-independent case. A mixture of direction-dependent and directionindependent terms is excluded when KamLAND data are added. In Sec. 2 we present the model and the neutrino oscillation probabilities. In Sec. 3 we discuss the constraints from atmospheric and long-baseline neutrino experiments. In Sec. 4 we discuss the constraints from solar neutrino experiments, and in Sec. 5 we discuss the combined constraints, including KamLAND. In Sec. 6 we present our conclusions. 


\section{Neutrino oscillations in the generalized bicycle model}

Neutrino oscillations occur due to eigen energy differences in $h_{\text {eff }}$ and the fact that the neutrino flavor eigenstates are not eigenstates of $h_{e f f}$. For massless neutrinos $p_{\mu}=(E,-E \hat{p})$, where $\hat{p}$ is the direction of neutrino propagation. Then for the generalized bicycle model

$$
h_{e f f}=\left(\begin{array}{ccc}
-2 c E & \frac{1}{\sqrt{2}} a \cos \Theta & \frac{1}{\sqrt{2}} a \cos \Theta \\
\frac{1}{\sqrt{2}} a \cos \Theta & 0 & 0 \\
\frac{1}{\sqrt{2}} a \cos \Theta & 0 & 0
\end{array}\right) \text {, }
$$

where

$$
\cos \Theta=\hat{p} \cdot \hat{n},
$$

i.e., $\Theta$ is the angle between the neutrino momentum and the preferred direction. From the diagonalization of $h_{e f f}$, there are two independent eigenenergy differences $\Delta_{j k}=E_{j}-E_{k}$,

$$
\Delta_{21}=\frac{m_{0}^{2}}{E_{0}^{2}}\left(\sqrt{E^{2}+E_{0}^{2} \cos ^{2} \Theta}+E\right), \quad \Delta_{32}=\frac{m_{0}^{2}}{E_{0}^{2}}\left(\sqrt{E^{2}+E_{0}^{2} \cos ^{2} \Theta}-E\right),
$$

where $m_{0}^{2}$ and $E_{0}$ are defined in terms of the Lorentz-invariance violating parameters by

$$
E_{0} \equiv \frac{a}{c}, \quad m_{0}^{2} \equiv \frac{a^{2}}{c},
$$

and the energy-dependent mixing angle is

$$
\sin ^{2} \theta=\frac{1}{2}\left[1-\frac{E}{\sqrt{E^{2}+E_{0}^{2} \cos ^{2} \Theta}}\right] .
$$

The off-diagonal oscillation probabilities are [5]

$$
\begin{aligned}
& P\left(\nu_{e} \leftrightarrow \nu_{\mu}\right)=P\left(\nu_{e} \leftrightarrow \nu_{\tau}\right)=2 \sin ^{2} \theta \cos ^{2} \theta \sin ^{2}\left(\Delta_{31} L / 2\right), \\
& P\left(\nu_{\mu} \leftrightarrow \nu_{\tau}\right)=\sin ^{2} \theta \sin ^{2}\left(\Delta_{21} L / 2\right)-\sin ^{2} \theta \cos ^{2} \theta \sin ^{2}\left(\Delta_{31} L / 2\right)+\cos ^{2} \theta \sin ^{2}\left(\Delta_{32} L / 2\right),
\end{aligned}
$$

where $\Delta_{31}=\Delta_{32}+\Delta_{21}$.

If $E_{0}^{2} \ll E^{2}$, i.e., $a^{2} \ll(c E)^{2}$, for atmospheric and long-baseline neutrinos, then $\sin ^{2} \theta \ll 1$, $\cos ^{2} \theta \simeq 1$ and the only appreciable oscillation is

$$
P\left(\nu_{\mu} \leftrightarrow \nu_{\tau}\right) \simeq \sin ^{2}\left(\Delta_{32} L / 2\right)
$$

where

$$
\Delta_{32} \simeq \frac{m_{0}^{2}}{2 E} \cos ^{2} \Theta
$$

Thus the oscillation amplitude is maximal, the effective mass-squared difference is

$$
\delta m_{e f f}^{2}=m_{0}^{2} \cos ^{2} \Theta
$$


and the energy dependence in this limit is the same as for conventional neutrino oscillations due to neutrino mass differences. Since the measured values for $\delta m_{\text {eff }}^{2}$ agree for atmospheric neutrinos and the K2K [8] and MINOS [9] long-baseline experiments, the effective $\cos ^{2} \Theta$ must also have similar values in all of these experiments.

\section{Atmospheric and long-baseline neutrinos}

\subsection{Directional dependence}

With the Earth's rotation axis chosen as the $\hat{Z}$ direction and the position of the detector given by $(\theta, \phi)$ in a standard spherical polar coordinate system (see Fig. 1), the neutrino direction can be written as

$$
\hat{p}=-\cos \beta \hat{r}+\sin \beta(-\sin \alpha \hat{\theta}+\cos \alpha \hat{\phi}),
$$

where $\mathbf{r}$ denotes the detector position, and the unit vectors $\hat{r}, \hat{\theta}$ and $\hat{\phi}$ point in the upward, southerly and easterly directions, respectively. The angle $\beta$ is the usual zenith angle $(\beta=0$ for a downward event) and $\alpha$ denotes the compass direction of the neutrino velocity projected on the plane tangent to the Earth's surface ( $\alpha=0$ for a neutrino going in the eastward direction). We take the preferred direction to be

$$
\hat{n}=\sin \xi \cos \chi \hat{X}+\sin \xi \sin \chi \hat{Y}+\cos \xi \hat{Z} .
$$

In our spherical polar coordinate system

$$
\begin{aligned}
\hat{n}= & {\left[\sin \xi \cos (\phi+\chi) \cos \theta_{L}+\cos \xi \sin \theta_{L}\right] \hat{r}+\left[\sin \xi \cos (\phi+\chi) \sin \theta_{L}-\cos \xi \cos \theta_{L}\right] \hat{\theta} } \\
& -\sin \xi \sin (\phi+\chi) \hat{\phi},
\end{aligned}
$$

where the usual angle spherical polar $\theta$ has been replaced by the latitude of the detector $\theta_{L}=\frac{\pi}{2}-\theta$. (positive for the northern hemisphere, negative for the southern hemisphere). The azimuthal angle $\phi$ is chosen so that $\phi=0$ corresponds to the preferred direction $\chi$, so that the angle $\chi$ may be dropped. The angular dependence in the oscillation formulas is then

$$
\begin{aligned}
\cos \Theta= & \cos \xi\left(\sin \beta \sin \alpha \cos \theta_{L}-\cos \beta \sin \theta_{L}\right) \\
& -\sin \xi \cos \phi\left(\sin \beta \sin \alpha \sin \theta_{L}+\cos \beta \cos \theta_{L}\right)-\sin \xi \sin \beta \cos \alpha \sin \phi .
\end{aligned}
$$

In Eq. (15), $\xi$ gives the orientation of the preferred axis with respect to the Earth's rotation axis, $\alpha$ (compass direction) and $\beta$ (zenith angle) relate to the neutrino direction, and $\phi$ depends on the time of the sidereal day ( $\phi=0$ when the detector is facing the preferred direction).

To help understand the complicated angular dependences in Eq. (15), we consider three special cases:

$$
\text { downward }(\beta=0) \quad: \quad \cos \Theta=-\left(\cos \xi \sin \theta_{L}+\sin \xi \cos \theta_{L} \cos \phi\right),
$$




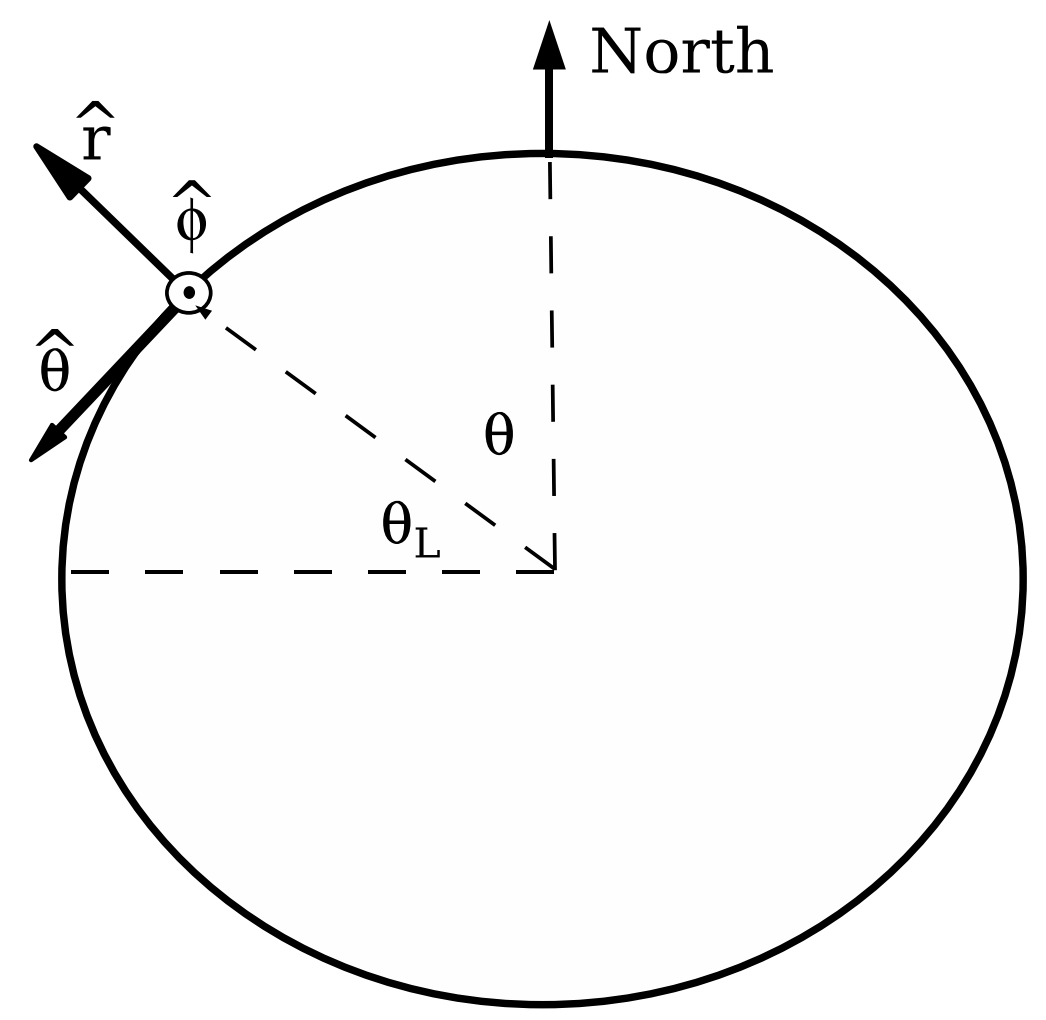

Figure 1: Detector position in atmospheric and long-baseline experiments. The angle $\theta_{L}$ is the latitude, while $\phi$ (not shown) measures the time of the sidereal day. The unit vectors $\hat{r}, \hat{\theta}, \hat{\phi}$ define the upward, southerly and easterly directions, respectively, for a neutrino event in the detector.

$$
\begin{aligned}
\operatorname{upward}(\beta=\pi) & : \cos \Theta=\cos \xi \sin \theta_{L}+\sin \xi \cos \theta_{L} \cos \phi, \\
\operatorname{horizontal}(\beta=\pi / 2) & : \quad \cos \Theta=\cos \xi \cos \theta_{L} \sin \alpha-\sin \xi\left(\sin \theta_{L} \cos \phi \sin \alpha+\sin \phi \cos \alpha\right) .
\end{aligned}
$$

Note that since only $\cos ^{2} \Theta$ appears in the oscillation formulas, the oscillation wavelengths for upward and downward events are the same.

\section{$3.2 \xi=0$}

If the preferred direction is aligned with the Earth's rotation axis, then $\xi=0$ and

$$
\cos ^{2} \Theta=\left(\sin \beta \sin \alpha \cos \theta_{L}-\cos \beta \sin \theta_{L}\right)^{2}
$$

Note that in this case $\Theta$ does not depend on time of day (measured by $\phi$ ). For accelerator experiments with relatively short baselines compared to the Earth's radius (such as K2K and MINOS), the neutrino path can be considered to be in the plane that is tangent to the Earth's surface, so that Eq. (18) applies and $\cos ^{2} \Theta=\sin ^{2} \alpha \cos ^{2} \theta_{L}$. Since the direction of the neutrino path in $\mathrm{K} 2 \mathrm{~K}$ is approximately given by $\alpha \simeq 174^{\circ}$ (slightly north of west), and the latitude of the Super-K detector 
is $\theta_{L} \simeq 36.3^{\circ}$,

$$
m_{0}^{2}=\frac{\delta m_{a}^{2}}{\sin ^{2} \alpha \cos ^{2} \theta_{L}} \simeq 0.4 \mathrm{eV}^{2}
$$

For MINOS, $\alpha \simeq 124^{\circ}$ and $\theta_{L} \simeq 48^{\circ}$, so that

$$
m_{0}^{2} \simeq 0.008 \mathrm{eV}^{2}
$$

which is nearly two orders of magnitude smaller than the value required to describe the K2K data. The reason $\mathrm{K} 2 \mathrm{~K}$ gives a much smaller value for $\cos ^{2} \Theta$ (and hence requires a much larger value for $m_{0}^{2}$ ) is that the neutrino path is nearly perpendicular to the Earth's rotation axis. Since the same $m_{0}^{2}$ applies to both, $\xi=0$ is excluded by a combination of the K2K and MINOS neutrino experiments.

We note that for upward or downward atmospheric neutrino events, $\cos ^{2} \Theta=\sin ^{2} \theta_{L}$, so $m_{0}^{2}=$ $\delta m_{a}^{2} / \sin ^{2} \theta_{L} \simeq 0.007 \mathrm{eV}^{2}$, which is very close to the value extracted from the MINOS data.

\section{$3.3 \xi \neq 0$}

If $\xi \neq 0$, then the preferred direction is not aligned with the Earth's rotation axis. For upward or downward atmospheric events there will be variation in $\cos ^{2} \Theta$ (and hence in $\delta m_{\text {eff }}^{2}$ ) over the sidereal period (see Eqs. (16) and (17)). At the time of the sidereal day when $\phi=0$ or $\pi$, there is always an extremum in $\cos ^{2} \Theta$. If $|\tan \xi|>\left|\tan \theta_{L}\right|$, then there are two more extrema at $\cos \phi=-\tan \theta_{L} / \tan \xi$. Thus there are two cases:

- For $|\tan \xi|<\left|\tan \theta_{L}\right|$, the only extrema of $\cos ^{2} \Theta$ occur at $\phi=0$ and $\pi$. Specifically, if $0<\xi<\theta_{L}$, then there is a minimum at $\phi=0$ and a maximum at $\phi=\pi$, and

$$
\sin ^{2}\left(\theta_{L}-\xi\right) \leq \cos ^{2} \Theta \leq \sin ^{2}\left(\theta_{L}+\xi\right)
$$

If $\pi-\theta_{L}<\xi<\pi$, then the positions of the maximum and minimum reverse, and

$$
\sin ^{2}\left(\xi+\theta_{L}\right) \leq \cos ^{2} \Theta \leq \sin ^{2}\left(\xi-\theta_{L}\right)
$$

- For $|\tan \xi|>\left|\tan \theta_{L}\right|$ (i.e., $\theta_{L}<\xi<\pi-\theta_{L}$ ), $\cos ^{2} \Theta=0$ when $\cos \phi=-\tan \theta_{L} / \tan \xi$ (which occurs twice a day) and there are maxima at $\phi=0$ and $\pi$ with $\cos ^{2} \Theta=\sin ^{2}\left(\xi \pm \theta_{L}\right)$. Therefore

$$
0 \leq \cos ^{2} \Theta \leq \max \left[\sin ^{2}\left(\xi-\theta_{L}\right), \sin ^{2}\left(\xi+\theta_{L}\right)\right]
$$

The solid curves in Fig. 2 show the maximum and minimum values of $\cos ^{2} \Theta$ versus $\xi$ for upward and downward atmospheric neutrinos. For $\theta_{L}<\xi<\pi-\theta_{L}$, there are always two times during the sidereal day when $\cos ^{2} \Theta=0$, and hence there are no oscillations for up/down events (since 


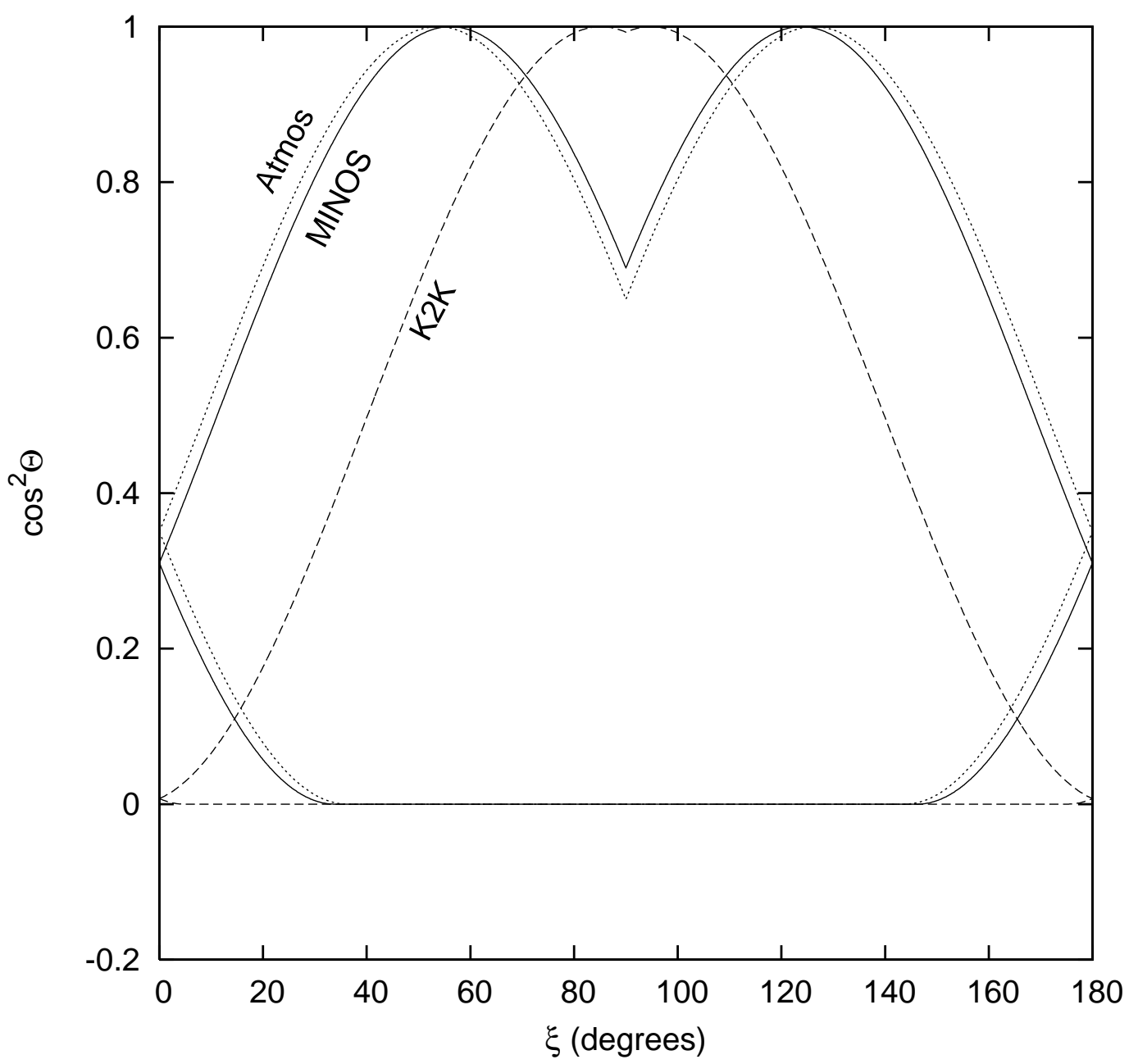

Figure 2: Maximum and minimum daily values for $\cos ^{2} \Theta$ versus $\xi$ for MINOS (solid curves), K2K (dashed) and Super-K up/down atmospheric (dotted) data. In regions with two different local maxima, the larger one is shown.

$\delta m_{\text {eff }}^{2}=m_{0}^{2} \cos ^{2} \Theta$ ). This effect might be evident in the Super-K data if it were binned according to sidereal time. For values of $\xi$ less than $\theta_{L}$ (or more than $\pi-\theta_{L}$ ), $\cos ^{2} \Theta$ is always finite, with the degree of modulation decreasing as $\xi \rightarrow 0$ (or $\pi$ ).

There is a similar situation for horizontal events, except that the critical angle that determines the number of extrema (and the values for the extrema) is $\gamma=\sin ^{-1}\left(\sin \alpha \cos \theta_{L}\right)$ instead of $\theta_{L}$. For $\mathrm{K} 2 \mathrm{~K}, \gamma \simeq 5^{\circ}$, and the minimum $\cos ^{2} \Theta$ is zero everywhere in the range $5^{\circ}<\xi<175^{\circ}$. For $0 \leq \xi \leq 5^{\circ}$ and $175^{\circ} \leq \xi \leq 180^{\circ}$, the minimum $\cos ^{2} \Theta$ is never larger than $\sin ^{2} 5^{\circ} \simeq 0.008$, so that there is always a time of day for $\mathrm{K} 2 \mathrm{~K}$ at which $\delta m_{\text {eff }}^{2}$ is suppressed and there are effectively no oscillations. The maximum and minimum $\cos ^{2} \Theta$ for K2K are also shown in Fig. 2, For MINOS, $\theta_{L}=47.8^{\circ}$ and the neutrino direction is approximately $\alpha=124^{\circ}$; then $\gamma=34^{\circ}$ and the MINOS daily ranges for $\cos ^{2} \Theta$ are almost identical to those for Super-K up/down atmospheric events (see 
Fig. (2).

For $\xi<\pi / 2$, the maximum value for $\delta m_{\text {eff }}^{2}$ in $\mathrm{K} 2 \mathrm{~K}$ is $m_{0}^{2} \sin ^{2}\left(\xi+\gamma_{K 2 K}\right)$, and for $\xi \leq \gamma_{M I N O S}$, the minimum value for $\delta m_{\text {eff }}^{2}$ in MINOS is $m_{0}^{2} \sin ^{2}\left(\xi-\gamma_{M I N O S}\right)$. For $\xi \leq 12^{\circ}$ there is no value of $m_{0}^{2}$ that gives $\delta m_{\text {eff }}^{2}$ within both allowed experimental ranges $\left(1.9 \times 10^{-3} \mathrm{eV}^{2} \leq \delta m^{2} \leq 3.5 \times 10^{-3} \mathrm{eV}^{2}\right.$ for $\mathrm{K} 2 \mathrm{~K}$ and $2.3 \times 10^{-3} \mathrm{eV}^{2} \leq \delta m^{2} \leq 3.4 \times 10^{-3} \mathrm{eV}^{2}$ for atmospheric neutrinos, at $90 \% \mathrm{C}$. L.) 1 Therefore, in an argument similar to the $\xi=0$ case, the predicted MINOS and K2K $\delta m_{\text {eff }}^{2}$ disagree for $\xi<12^{\circ}$, in contradiction with data, so that these values are excluded. For $12^{\circ}<\xi<90^{\circ}$, there are always two times during the sidereal day when $\cos ^{2} \Theta=0$ for $\mathrm{K} 2 \mathrm{~K}$, and there are no oscillations. For $12^{\circ}<\xi<36^{\circ}$, atmospheric up/down events should show a significant modulation of $\delta m_{e f f}^{2}$, and for $36^{\circ}<\xi<90^{\circ}$ there are always two times during the sidereal day when $\cos ^{2} \Theta=0$ for atmospheric up/down events. Similar comments can be made for the range $\pi / 2<\xi<\pi$.

The results for K2K, MINOS and up/down atmospheric neutrinos may be summarized as follows:

- The range $0<\xi<12^{\circ}$ (and by similar arguments, $168^{\circ}<\xi<180^{\circ}$ ) is excluded by a comparison of the measured $\delta m^{2}$ values in MINOS and $\mathrm{K} 2 \mathrm{~K}$ data.

- For $12^{\circ}<\xi<168^{\circ}$, there are always two times during the sidereal day when K2K should have no oscillations, i.e., no suppression of events relative to expectation. Up/down atmospheric neutrinos always have a significant modulation of $\delta m_{\text {eff }}^{2}$, and for $36^{\circ}<\xi<144^{\circ}$ there are always two times during the sidereal day when up/down atmospheric neutrinos should also have no suppression.

For horizontal atmospheric neutrino events $(\beta=\pi / 2), \cos \Theta$ is given by Eq. (18); the daily fluctuations then depend on the compass direction of the event, $\alpha$. Super-K has measured the compass dependence [10] and found agreement with an east-west asymmetry due to the Earth's magnetic field, plus oscillations. Any additional compass dependence must not be too large to remain consistent with the data. Table 1 shows $\cos ^{2} \Theta$ for some typical values of $\alpha$ and $\phi$. The direction dependence would not enhance or suppress the east-west difference, but could enhance or suppress oscillations along the east/west direction compared to north/south. Furthermore, enhancements could change to suppression (and vice versa) during the sidereal period. A detailed analysis would be needed to determine the compass-direction dependence for horizontal atmospheric neutrino events.

\footnotetext{
${ }^{1}$ This is approximately equal to the region where the $\cos ^{2} \Theta$ values do not overlap in Fig. 2: the difference is due to the slightly different ranges for $\delta m^{2}$ in the two experiments.
} 
Table 1: Values of $\cos ^{2} \Theta$ for various values of $\alpha$ and $\phi$.

\begin{tabular}{|l|cccc|}
\hline$\alpha$ & $\phi=0$ & $\phi=\pi / 2$ & $\phi=\pi$ & $\phi=3 \pi / 2$ \\
\hline $0, \pi(\mathrm{E}, \mathrm{W})$ & 0 & $\sin ^{2} \xi$ & 0 & $\sin ^{2} \xi$ \\
$\pi / 2, \frac{3 \pi}{2}(\mathrm{~N}, \mathrm{~S})$ & $\cos ^{2}\left(\xi-\theta_{L}\right)$ & $\cos ^{2} \xi \cos ^{2} \theta_{L}$ & $\cos ^{2}\left(\xi+\theta_{L}\right)$ & $\cos ^{2} \xi \cos ^{2} \theta_{L}$ \\
\hline
\end{tabular}

\section{Solar neutrinos}

\subsection{Directional dependence}

In a coordinate system $\left(X^{\prime}, Y^{\prime}, Z^{\prime}\right)$ where the $Z^{\prime}$ axis is perpendicular to the Earth's orbital plane (the ecliptic plane), the direction of neutrino propagation may be written as (see Fig. 3)

$$
\hat{p}=\cos \psi \hat{X}^{\prime}+\sin \psi \hat{Y}^{\prime},
$$

where $\psi$ gives the position of the Earth in its orbit $(\psi=0$ at the vernal equinox, $\psi=\pi / 2$ at the summer solstice, etc.). The equatorial coordinates are related to the ecliptic coordinates via rotation by an angle $\eta \simeq 23^{\circ}$ about the $X^{\prime}$ axis, where $\eta$ is the tilt of the Earth's rotation axis from the perpendicular to the ecliptic (see Fig. 3). Then in the celestial equatorial frame the direction of propagation for solar neutrinos is

$$
\hat{p}=\cos \psi \hat{X}+\sin \psi \cos \eta \hat{Y}-\sin \psi \sin \eta \hat{Z},
$$

and therefore

$$
\cos \Theta=\hat{p} \cdot \hat{n}=\cos \psi \cos \chi \sin \xi+\sin \psi(\sin \chi \sin \xi \cos \eta-\cos \xi \sin \eta)
$$

Note that $\cos \Theta$ for solar neutrinos is independent of detector latitude $\left(\theta_{L}\right)$ and time of day $(\phi)$.

\subsection{Oscillation probability}

In matter there is an additional term in the hamiltonian due to coherent forward scattering of $\nu_{e}$ 's with electrons in matter, so that $-2 c E$ in the upper left element of $h_{\text {eff }}$ is replaced by $-2 c E+$ $\sqrt{2} G_{F} N_{e}$ and the mixing angle in Eq. (6) is then given by

$$
\sin ^{2} \theta=\frac{1}{2}\left[1-\frac{c E-G_{F} N_{e} / \sqrt{2}}{\sqrt{\left(c E-G_{F} N_{e} / \sqrt{2}\right)^{2}+a^{2} \cos ^{2} \Theta}}\right],
$$

where $N_{e}$ is the electron number density. For adiabatic propagation in the sun the solar neutrino oscillation probability is

$$
P\left(\nu_{e} \rightarrow \nu_{e}\right)=\cos ^{2} \theta \cos ^{2} \theta_{0}+\sin ^{2} \theta \sin ^{2} \theta_{0},
$$



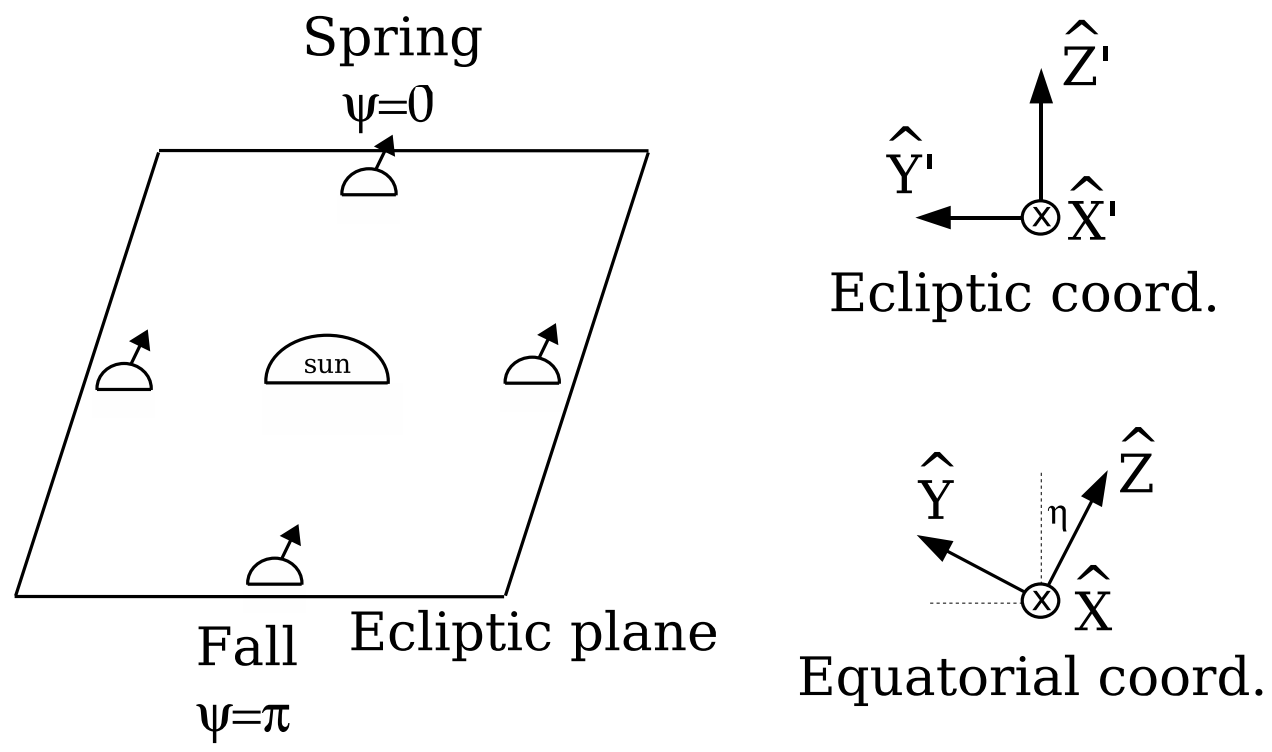

Figure 3: Position of the Earth in the ecliptic plane. The arrows represent the Earth's rotation axis and $\psi=0$ corresponds to the vernal equinox. The orientation of the celestial equatorial coordinate system relative to the ecliptic coodinate system is also shown.

where $\theta_{0}$ is the mixing angle at the creation point in the sun (with electron number density $N_{e}^{0} \simeq$ $90 N_{A} / \mathrm{cm}^{3}$ ) and $\theta$ is the mixing angle in vacuum. $P \rightarrow \frac{1}{2}$ at low energies 2 There is a minimum in $P$ at

$$
E_{\min }=\frac{G_{F} N_{e}^{0}}{2 \sqrt{2} c}
$$

with minimum value

$$
P_{\text {min }}\left(\nu_{e} \rightarrow \nu_{e}\right)=\frac{4 a^{2} \cos ^{2} \Theta}{8 a^{2} \cos ^{2} \Theta+\left(G_{F} N_{e}^{0}\right)^{2}}<\frac{1}{2},
$$

where $\cos \Theta$ is given by Eq. (27). At $E=2 E_{\min }$ there is a resonance and the probablity is $\frac{1}{2}$, and for $E>2 E_{\min }$ the probability increases monotonically, with limiting value unity as $E \rightarrow \infty$. The angle $\Theta$ depends on the time of year; averaging over $\psi$ gives

$$
\left\langle P_{\min }\left(\nu_{e} \rightarrow \nu_{e}\right)\right\rangle=\frac{1}{2}\left[1-\frac{G_{F} N_{e}^{0}}{\sqrt{\left(G_{F} N_{e}^{0}\right)^{2}+8 a^{2} D^{2}}}\right],
$$

where

$$
D^{2} \equiv \cos ^{2} \chi \sin ^{2} \xi+(\sin \chi \sin \xi \cos \eta-\cos \xi \sin \eta)^{2}
$$

If the probability minimum lies in the middle of the ${ }^{8} \mathrm{~B}$ solar neutrino region, then $\left\langle P_{\min }\right\rangle$ in Eq. (32) will give the approximate survival probability of the ${ }^{8} \mathrm{~B}$ neutrinos.

\footnotetext{
${ }^{2}$ The actual solar neutrino oscillation probability at low energies is closer to 0.7 [1]. However, if an additional $a_{e e}^{T T}$ term is included in $h_{e f f}$, then the low energy probability can be fit to the higher value, at the expense of adding a fifth parameter to the model.
} 
The formulas used above for the solar neutrino probability assumed adiabatic propagation. It can be shown that the propagation is adiabatic except close to the two times during the year where $\cos \Theta=0$ :

$$
\psi=-\sin ^{-1}\left(\frac{\cos \chi \sin \xi}{D}\right) \quad \text { and } \quad \pi-\sin ^{-1}\left(\frac{\cos \chi \sin \xi}{D}\right)
$$

this was also pointed out in Ref. [5] for the special case $\xi=0$. To include the effects of nonadiabatic propagation, Eq. (29) must be modified to

$$
P\left(\nu_{e} \rightarrow \nu_{e}\right)=\frac{1}{2}\left[1+\left(1-2 P_{x}\right)\left(\cos ^{2} \theta \cos ^{2} \theta_{0}+\sin ^{2} \theta \sin ^{2} \theta_{0}\right)\right],
$$

where $P_{x}$ is the level-crossing transition probability,

$$
P_{x}=e^{-\pi \gamma_{r} / 2},
$$

and $\gamma_{r}$ is the adiabaticity of the transition at the level-crossing resonance. For our Hamiltonian

$$
\gamma_{r}=\frac{2 \sqrt{2} a^{2} \cos ^{2} \Theta}{G_{F}\left|d N_{e} / d L\right|_{r}}
$$

where $\left|d N_{e} / d L\right|_{r}$ is the rate of change of $N_{e}$ at the resonance. At $E_{\text {min }}$ the probability becomes

$$
P_{\text {min }}\left(\nu_{e} \rightarrow \nu_{e}\right)=\frac{4 a^{2} \cos ^{2} \Theta}{8 a^{2} \cos ^{2} \Theta+\left(G_{F} N_{e}^{0}\right)^{2}}+\frac{\left(G_{F} N_{e}^{0}\right)^{2}}{\left(G_{F} N_{e}^{0}\right)^{2}+8 a^{2} \cos ^{2} \Theta} P_{x}
$$

where the first term on the right-hand side is the adiabatic contribution and the second term the nonadiabatic correction. Propagation is nonadiabatic when $\gamma_{r}$ is small, which occurs when $a^{2} \cos ^{2} \Theta$ is small. For the parameter ranges of interest we find that $8 a^{2} \cos ^{2} \Theta \ll\left(G_{F} N_{e}^{0}\right)^{2}$ in the regions where $P_{x}$ is nonnegligible, so that the probability reduces to

$$
P\left(\nu_{e} \rightarrow \nu_{e}\right) \simeq \frac{4 a^{2} \cos ^{2} \Theta}{8 a^{2} \cos ^{2} \Theta+\left(G_{F} N_{e}^{0}\right)^{2}}+P_{x}
$$

From Eqs. (36), (37) and (39) we see that the survival probability goes to unity when $\cos \Theta=0$. The probability is shown in Fig. 4 versus $\psi$ using both the adiabatic and nonadiabatic formulas; they differ substantially only near the values of $\psi$ given by Eq. (34).

\subsection{Constraints from solar data}

In order to fit the solar neutrino data, $\left\langle P_{\min }\right\rangle$ must match the measured probability for the ${ }^{8} \mathrm{~B}$ neutrinos, i.e., $\left\langle P_{\min }\right\rangle \simeq 0.34$ (we use the ratio of $\mathrm{CC}$ to NC rates in SNO 12 to avoid complications due to theoretical uncertainties in the solar neutrino spectrum). Since there is no apparent energy dependence in the ${ }^{8} \mathrm{~B}$ oscillation probability, the minimum must occur near the middle of the ${ }^{8} \mathrm{~B}$ 


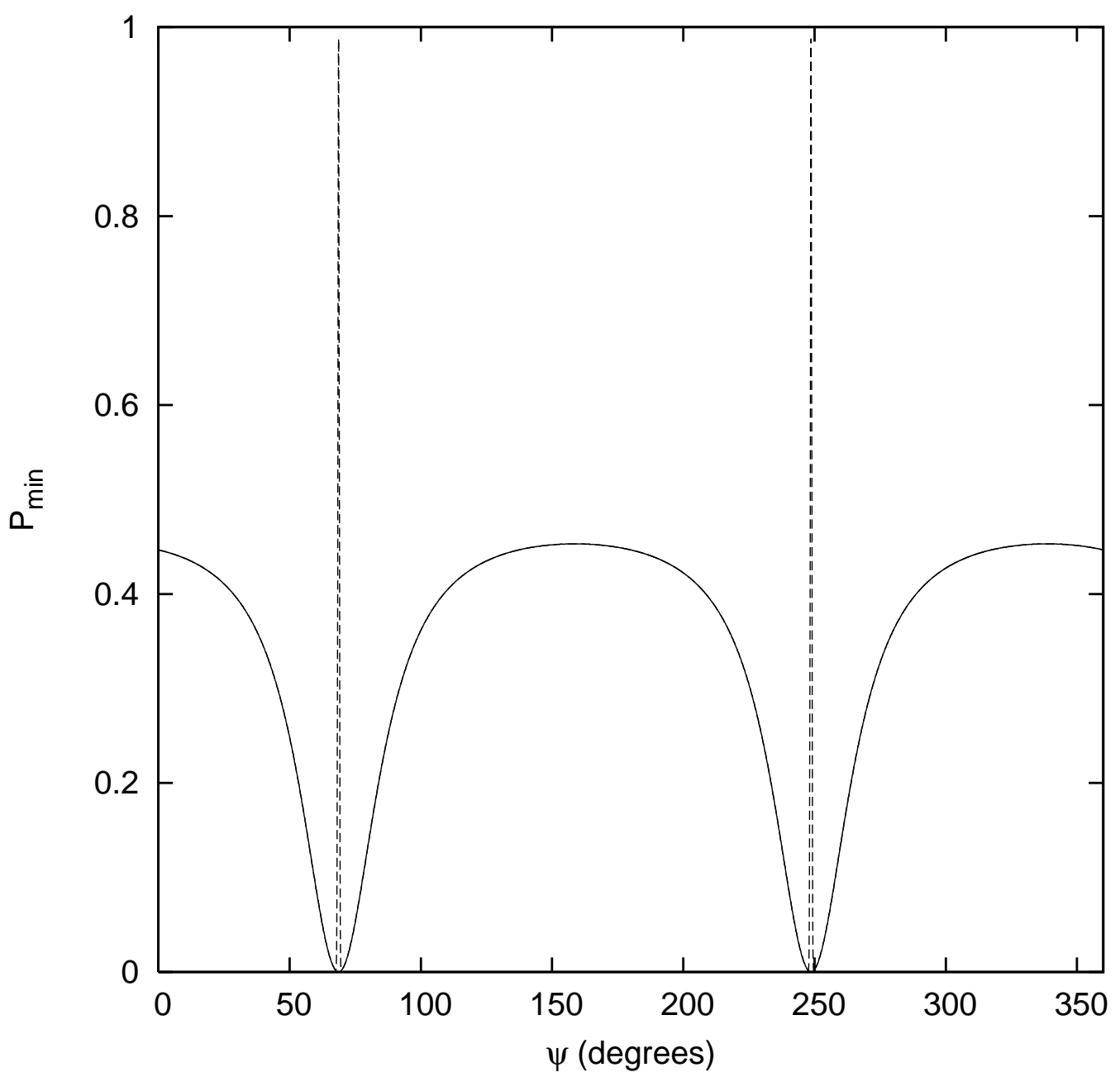

Figure 4: Representative solar neutrino survival probability at $E_{\min }$ using the adiabatic (Eq. (31), solid curve) and nonadiabatic (Eq. (39), dashed) formulas, shown versus the time of year (measured by $\psi$ ). The parameters for this example are $a=7 \times 10^{-12} \mathrm{eV}, \xi=45^{\circ}$ and $\chi=0$, which give $\left\langle P_{\min }\right\rangle \simeq 0.34$. The two formulas differ only close to the values of $\psi$ where $\cos \Theta=0$.

spectrum $\left(E_{\min } \simeq 10 \mathrm{MeV}\right)$, so that probabilities at either end of the spectrum are not much larger than in the middle. This results in the two constraints (from Eqs. (301) and (32))

$$
\begin{aligned}
c & \simeq \frac{G_{F} N_{e}^{0}}{2 \sqrt{2} E_{\min }}=1.7 \times 10^{-19} \\
a D & \simeq \sqrt{2} G_{F} N_{e}^{0} \frac{\sqrt{\left\langle P_{\min }\right\rangle\left(1-\left\langle P_{\min }\right\rangle\right)}}{\left(1-2\left\langle P_{\min }\right\rangle\right)}=5.0 \times 10^{-12} \mathrm{eV},
\end{aligned}
$$

where Eq. (41) uses the adiabatic expression for $\left\langle P_{\min }\right\rangle$. Since Eq. (40) depends only on the initial density for ${ }^{8} \mathrm{~B}$ neutrinos and the central energy of the SNO spectrum, we will use this result for $c$ throughout the rest of this paper.

We note that although the value of $a$ required to fit $\left\langle P_{\min }\right\rangle$ depends on the value of $D$ (which in 
turn depends on the preferred-direction parameters $\xi$ and $\chi$ ), the product $a D$ is fixed by Eq. (41), and the oscillation probability versus time will always be identical to that shown in Fig. 4, except for a possible shift in phase and the corrections for the two nonadiabatic spikes. This can be understood by rewriting the adiabatic probability in Eq. (31) as

$$
P_{\text {min }}=\frac{4 a^{2} D^{2} \sin ^{2}(\psi+\delta)}{8 a^{2} D^{2} \sin ^{2}(\psi+\delta)+\left(G_{F} N_{e}^{0}\right)^{2}},
$$

where

$$
\tan \delta \equiv \frac{\sin \xi \cos \chi}{\sin \chi \sin \xi \cos \eta-\cos \xi \sin \eta}
$$

Thus the time variation of $P_{\min }$ has the same shape and maximum and minimum values when $a D$ is held fixed.

The measured solar neutrino survival probability does not exhibit much variation throughout the year. The SNO collaboration has tested their solar neutrino data for periodicities [13] and found a variation during the year that is consistent with the $1 / r^{2}$ dependence of the flux as the Earth's distance from the sun varies. The uncertainties in the rate are of order $3-5 \%$, so there is little room for any additional annual variation. The SNO periodicity data sample includes all of their solar neutrino data in both the $\mathrm{D}_{2} \mathrm{O}$ phase and salt phase, and combines events from chargecurrent (CC), neutral-current (NC), electron scattering (ES) and backgrounds (B). They measured the relative event rate versus time of year, normalized to the mean rate, i.e.,

$$
R=\frac{N_{N C}^{0}+N_{C C}^{0} P+N_{E S}^{0}[P+r(1-P)]+N_{B}^{0}}{N_{N C}^{0}+N_{C C}^{0}\langle P\rangle+N_{E S}^{0}[\langle P\rangle+r(1-\langle P\rangle)]+N_{B}^{0}},
$$

where $P$ is the oscillation probability, $N_{i}^{0}$ is the number of events expected without oscillations, $r$ is the ratio of the $\mathrm{NC}$ to $\mathrm{CC}$ cross sections and angle brackets indicate mean values. For $\left\langle P_{\min }\right\rangle=$ 0.34 , the bicycle model with directional dependence predicts $R$ should vary between 0.42 and 1.19 throughout the year. Since the SNO measurement of $R$ varies by at most $5 \%$ at any time during the year, the pure direction-dependent case clearly cannot fit the SNO periodicity test while simultaneously reproducing the correct average survival probabilty.

To verify this quantitatively we have searched the $a, \xi$ and $\chi$ parameter space via Monte Carlo, using the twelve bins of the SNO periodicity data and the SNO average probability $(0.34 \pm 0.03$, from the $\mathrm{CC}$ to $\mathrm{NC}$ ratio). We have used the appropriate weighting of run times and $\mathrm{D}_{2} \mathrm{O} / \mathrm{salt}$ phase for each bin, and used Eq. (39) for the oscillation probablity, which includes the nonadiabatic part. The nonadiabatic spikes appreciably affect bin-averaged probabilities only in the bins where they occur, and then by order 0.05 or less. In Fig. 5 we show the SNO periodicity data plus the best fit when varying over $a, \xi$ and $\chi$ when $\langle P\rangle$ is constrained to lie within $1 \sigma$ of the central value. Allowing $\langle P\rangle$ to lie outside the $1 \sigma$ range can improve the $\chi^{2}$, but in all cases the $\chi^{2}$ per degree of freedom (DOF) is such that the probablity that the model describes the data is $2 \times 10^{-8}$ or 


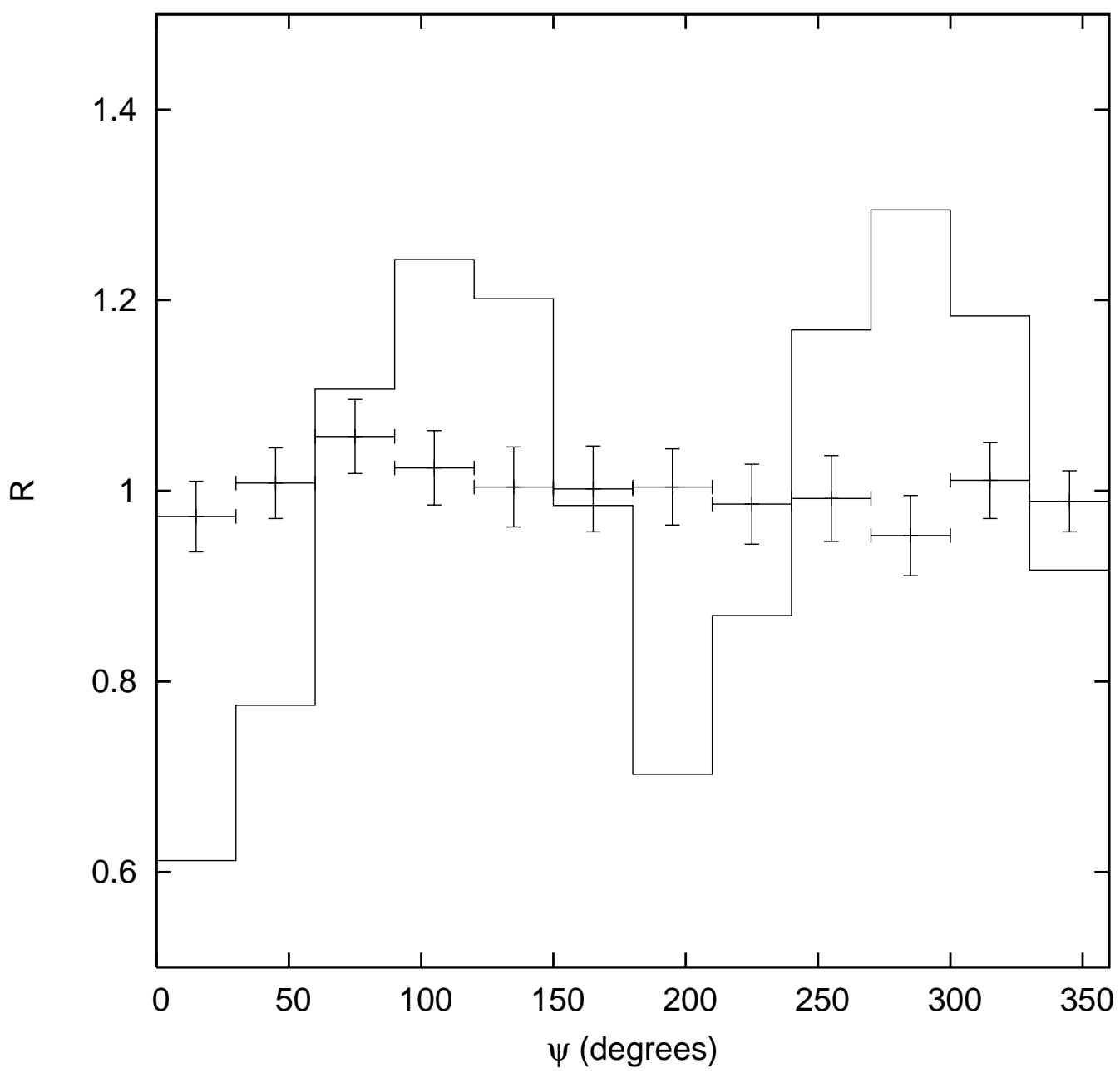

Figure 5: Best-fit prediction for $R$ in Eq. (44) for the ${ }^{8} \mathrm{~B}$ neutrinos (solid curve) and the SNO measured value for $R$ (data points) shown versus time of year (measured by $\psi$ ). Both the SNO data and the model predictions are averaged over each bin, and the SNO data has been corrected for the $1 / r^{2}$ variation due to the changing Earth-Sun distance. The prediction for $\langle P\rangle$ has also been constrained to lie witin $1 \sigma$ of the SNO central value. The model parameters for the best fit are $a=1.96 \times 10^{-12} \mathrm{eV}^{2}, \xi=43^{\circ}$ and $\chi=298^{\circ}$, with $\chi^{2} / D O F=361 / 10$.

less. The best fit has very little annual variation, but $\langle P\rangle \simeq 0.21$. Therefore we conclude that the generalized direction-dependent bicycle model is strongly ruled out solely by the solar neutrino data. 


\section{Combined constraints}

\subsection{Adding a direction independent term}

Since the pure direction-dependent case is ruled out, we now generalize the model to include both direction-independent as well as direction-dependent terms in the off-diagonal elements of $h_{e f f}$. This increases the number of parameters in the model to five. If we define $\left(a_{L}\right)_{e \mu}^{\mu}=\left(a_{L}\right)_{e \tau}^{\mu}=$ $(a \cos \rho, a \sin \rho \hat{n} / \sqrt{2})$, where $\hat{n}$ is again the preferred direction, then $\cos \Theta$ should be replaced by $\cos \rho+\sin \rho \cos \Theta$ in our previous formulas. The parameter $\rho$ determines the amount of direction dependence: $\rho=\pi / 2$ or $3 \pi / 2$ corresponds to the pure direction-dependent case we discussed before, while $\rho=0$ or $\pi$ corresponds to no direction dependence.

For a given preferred direction (fixed $\xi$ and $\chi$ ), the parameters $c$ and $a$ are determined from the solar neutrino data using Eqs. (30) and (31), after the substitution $\cos \Theta \rightarrow \cos \rho+\sin \rho \cos \Theta$ is made. Then using Eqs. (5) and (11), $\delta m_{\text {eff }}^{2}$ for long-baseline and atmospheric neutrinos may be written as

$$
\delta m_{e f f}^{2}=\frac{a^{2}}{c}(\cos \rho+\sin \rho \cos \Theta)^{2} .
$$

It is convenient to rewrite $\cos \Theta$ as

$$
\cos \Theta=D \sin (\psi+\delta)
$$

where $\delta$ is defined in Eq. (43). Integrating $P_{\min }$ in the modified Eq. (31) over $\psi$, leads to

$$
\left\langle P_{\min }\right\rangle=\frac{1}{2}\left[1-\frac{G_{F} N_{e}^{0}}{\sqrt{2} S^{2}} \sqrt{S^{2}+8 a^{2}\left(D^{2} \sin ^{2} \rho-\cos ^{2} \rho\right)+\left(G_{F} N_{e}^{0}\right)^{2}}\right],
$$

for adiabatic neutrinos, where

$$
S^{2}=\sqrt{64 a^{4}\left(D^{2} \sin ^{2} \rho-\cos ^{2} \rho\right)^{2}+16 a^{2}\left(G_{F} N_{e}^{0}\right)^{2}\left(D^{2} \sin ^{2} \rho+\cos ^{2} \rho\right)+\left(G_{F} N_{e}^{0}\right)^{4}} .
$$

\subsection{No direction dependence}

For the pure direction-independent case $(\rho=0$ or $\pi), \delta m_{\text {eff }}^{2}=m_{0}^{2}=a^{2} / c$ for atmospheric and long-baseline neutrinos and $P_{\min }$ for ${ }^{8} \mathrm{~B}$ solar neutrinos is given simply by Eq. (31); for $P_{\min }=0.34$,

$$
a=\sqrt{\frac{P_{\text {min }}}{2\left(1-2 P_{\text {min }}\right)}} \frac{G_{F} N_{e}^{0}}{2} \simeq 2.5 \times 10^{-12} \mathrm{eV},
$$

and the prediction from the solar neutrino data is $\delta m_{\text {eff }}^{2}=3.6 \times 10^{-5} \mathrm{eV}^{2}$ for atmospheric and longbaseline neutrinos, which is clearly in contradiction with the data. Therefore, the pure directionindependent case is ruled out by the combined data. 


\subsection{Mixed case}

For a mixture of direction-dependent and direction-independent terms in $h_{e f f}$, a fit must be done to the solar data to determine an allowed region in parameter space, and then the predictions for $\delta m_{\text {eff }}^{2}$ in long-baseline experiments can be compared to data. To fit the solar data we take the 12 bins from the SNO periodicity data sample for the relative rate $R$ and add the additional constraint that the average oscillation probability must be $P=0.34 \pm 0.03$, as described in the previous section. As before, we fix the value of $c$ to that given in Eq. (40), and vary over the parameters $\xi, \chi, \rho$ and $a$ with a Monte Carlo. The $99 \%$ C. L. allowed regions are determined by restricting the $\chi^{2} / D O F$ to be less than 2.4 for nine DOF (there are thirteen data points and four parameters). The best fit to the SNO data has $a=3.0 \times 10^{-12} \mathrm{eV}^{2}, \xi=21^{\circ}, \chi=94^{\circ}$ and $\rho=114^{\circ}$, with $\chi^{2} / D O F=4.84 / 9$.

Predictions for $\delta m_{\text {eff }}^{2}$ can then be made for K2K and MINOS. Since $\delta m_{\text {eff }}^{2}$ depends on cos $\Theta$, it will vary during the sidereal day for $\xi \neq 0$, with ranges depending on $\xi$ as shown in Fig. 2. The strictest constraints come from $\mathrm{K} 2 \mathrm{~K}$; maximum possible values of $\delta m_{\text {eff }}^{2}$ in $\mathrm{K} 2 \mathrm{~K}$ are shown versus $\xi$ in Fig. 6.

In most all cases the maximum possible $\delta m_{\text {eff }}^{2}$ can never be in the experimentally measured range $1.9 \times 10^{-3} \leq \delta m^{2} \leq 3.5 \times 10^{-3} \mathrm{eV}^{2}$. Only a small region near $\xi \simeq \eta=23^{\circ}$ or $\pi-\eta \simeq 157^{\circ}$ can give a large enough value of $\delta m_{\text {eff }}^{2}$. This allowed region is also characterized by $\rho \simeq \pi / 2$ or $3 \pi / 2$ and $\chi \simeq \pi / 2$ or $3 \pi / 2$, such that $|D \sin \rho| \ll|\cos \rho|$, and values of $a \geq 3 \times 10^{-11} \mathrm{eV}$.

As evident from Eq. (46), this results in $\cos \Theta \simeq 0$ for solar neutrinos (i.e., the preferred direction is nearly perpendicular to the ecliptic plane), so that the directional dependence for solar neutrinos is minimal, even though the direction-dependent coefficient $\sin \rho$ is much larger than the directionindependent coefficient $\cos \rho$. For atmospheric and long-baseline neutrinos this fortuitous situation does not occur and the direction-dependent piece is sizable, with daily variations of $\cos ^{2} \Theta$ given by Fig. 2. Therefore the case with a mixture of direction-dependent and direction-independent terms is severely constrained, and there is a strong variation of $\delta m_{e f f}^{2}$ for atmospheric and long-baseline neutrinos during the sidereal day for the allowed solutions.

\subsection{KamLAND}

For reactor neutrinos with both direction-dependent and independent terms, from Eqs. (44)-(8) we have

$$
P\left(\bar{\nu}_{e} \rightarrow \bar{\nu}_{e}\right)=1-\sin ^{2} 2 \theta \sin ^{2}\left(\frac{1}{2} \Delta_{31} L\right)
$$

where

$$
\Delta_{31}=2 \sqrt{(c E)^{2}+a^{2}(\cos \rho+\sin \rho \cos \Theta)^{2}},
$$




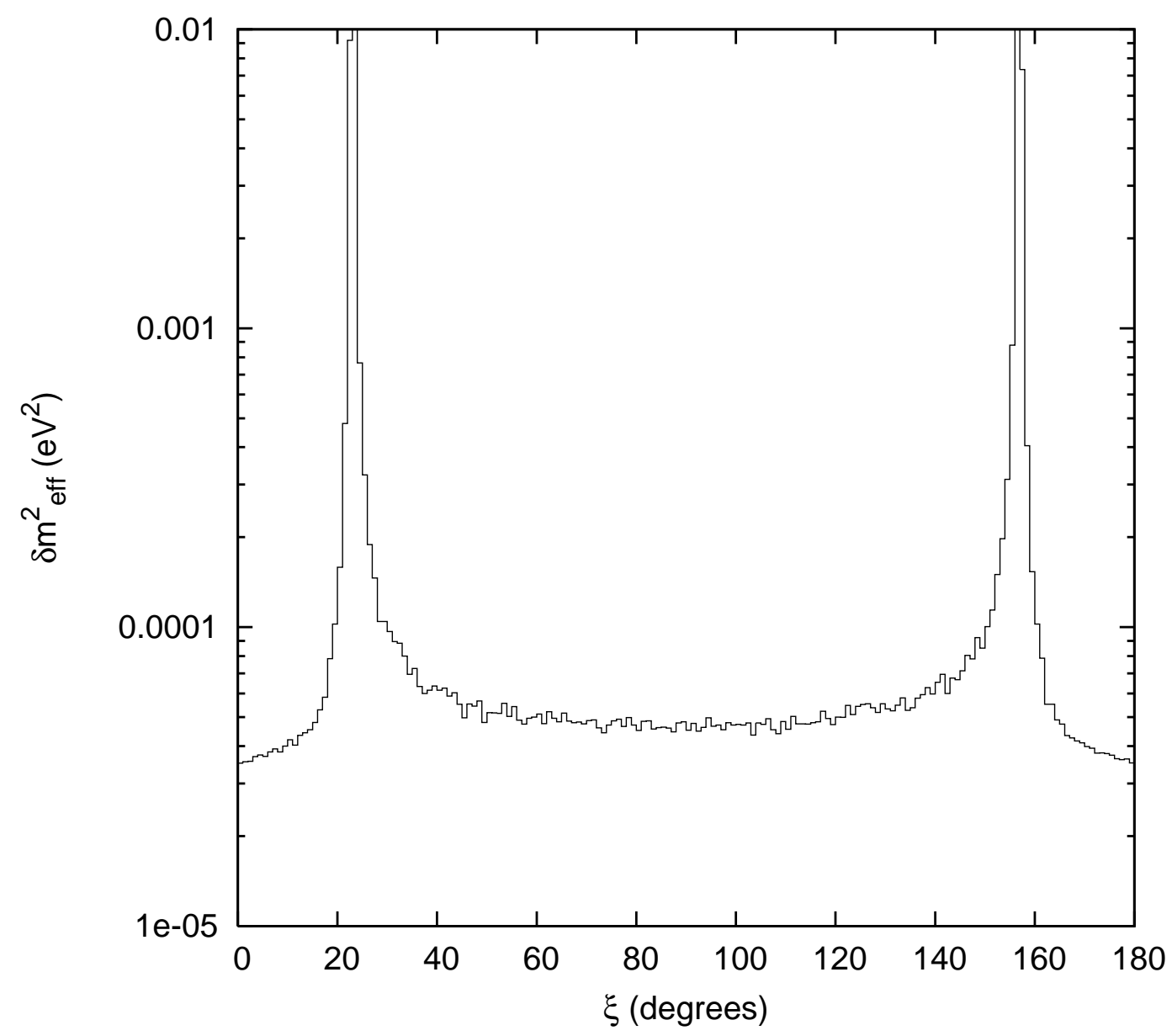

Figure 6: Maximum value of $\delta m^{2}$ in $\mathrm{K} 2 \mathrm{~K}$ allowed at $99 \%$ C. L. by model parameters consistent with the solar $R$ and $\langle P\rangle$ data, shown versus the preferred direction $\xi$.

$$
\sin ^{2} 2 \theta=1-\frac{(c E)^{2}}{(c E)^{2}+a^{2}(\cos \rho+\sin \rho \cos \Theta)^{2}}
$$

and $\cos \Theta$ is given by Eq. (18). For the values of the parameters that fit solar data and give a large enough $\delta m_{\text {eff }}^{2}$ for long-baseline neutrinos, $(c E)^{2} \ll a^{2}$ and $\sin ^{2} 2 \theta \simeq 1$ at reactor neutrino energies, except possibly for the brief time of day when $\cos \rho+\sin \rho \cos \Theta \rightarrow 0$.

As discussed in Sec. 3, given $\gamma=\sin ^{-1}\left(\sin \alpha \cos \theta_{L}\right)$, where $\alpha$ is the compass direction of the incoming neutrino and $\theta_{L}$ the latitude of the detector, the maximum and minimum values for $\cos ^{2} \Theta$ during the sidereal day are given by Eqs. (22)-(24) with $\theta_{L}$ replaced by $\gamma$. Then for the parameter ranges found above, it is not hard to show that for all of the reactors contributing to the KamLAND signal, the oscillation argument varies over many cycles during the day, so that the oscillation probability is close to 0.5 , regardless of neutrino energy. Thus the bicycle model gives a suppression in KamLAND that is nearly independent of energy, contrary to the KamLAND data [14, which excludes an energy-independent suppression at 99.6\% C. L. 
We have verified this result numerically using typical solar/atmosperic/long-baseline solutions, averaging over the sidereal day, and summing over individual reactor contributions - the suppression varies by at most 0.02 over the range $2.5 \leq E \leq 6 \mathrm{MeV}$ which supplies the bulk of the KamLAND data. The average survival probability of the bicycle model solutions is at most about 0.55 , well below the measured KamLAND value of $P=0.658 \pm 0.044 \pm 0.047$. Therefore the bicycle model with a mixture of direction-dependent and direction-independent terms is also excluded.

\section{Conclusions}

We have shown that the generalized five-parameter bicycle model with Lorentz-invariance violation and no neutrino masses can be ruled out by a combination of solar, long-baseline and reactor neutrino data. The pure direction-dependent case is ruled out because it gives a large annual variation in the oscillation probability for ${ }^{8} \mathrm{~B}$ solar neutrinos, at odds with SNO periodicity data. The pure direction-independent case is ruled out because the values of the parameters required to fit the SNO data predict a value of $\delta m^{2}$ in long-baseline experiments that is too small by nearly two orders of magnitude. Having a mixture of direction-dependent and direction-independent terms in the off-diagonal elements of $h_{e f f}$ is excluded when KamLAND is added to a combination of solar and long-baseline data.

Although the five-parameter bicycle model cannot fit all of the data, the full $h_{\text {eff }}$ with Lorentznoninvariant oscillations of massless neutrinos has 160 parameters [5], and a comprehensive comparison with data is impractical. However, it is clear that any direction dependence will encounter severe constraints, including variations during the sidereal day which were not pursued in this paper. Restricting $h_{e f f}$ to only direction-independent terms reduces the number of Lorentz-noninvariant parameters to 16 [5]. Even then, as our analysis of the direction-independent bicycle model suggests, finding a set of parameters that would simultaneously fit solar, atmospheric, long-baseline and reactor data will be difficult at best.

\section{Acknowledgments}

We thank A. Kostelecky for useful discussions. We also thank the Aspen Center for Physics for hospitality during the completion of this work. This research was supported by the U.S. Department of Energy under Grant Nos. DE-FG02-95ER40896, DE-FG02-01ER41155, and DE-FG02-04ER41308, by the NSF under CAREER Award No. PHY-0544278, and by the Wisconsin Alumni Research Foundation. 


\section{References}

[1] See e.g., V. Barger, D. Marfatia and K. Whisnant, Int. J. Mod. Phys. E 12, 569 (2003) arXiv:hep-ph/0308123]; S. Pakvasa and J. W. F. Valle, Proc. Indian Natl. Sci. Acad. 70A, 189 (2004) arXiv:hep-ph/0301061].

[2] A. Aguilar et al. [LSND Collaboration], Phys. Rev. D 64, 112007 (2001) arXiv:hep-ex/0104049.

[3] S. R. Coleman and S. L. Glashow, Phys. Rev. D 59, 116008 (1999) arXiv:hep-ph/9812418.

[4] V. D. Barger, S. Pakvasa, T. J. Weiler and K. Whisnant, Phys. Rev. Lett. 85, 5055 (2000) arXiv:hep-ph/0005197.

[5] V. A. Kostelecky and M. Mewes, Phys. Rev. D 70, 031902 (2004) arXiv:hep-ph/0308300.

[6] V. A. Kostelecky and M. Mewes, Phys. Rev. D 69, 016005 (2004) arXiv:hep-ph/0309025.

[7] T. Katori, A. Kostelecky and R. Tayloe, Phys. Rev. D 74, 105009 (2006) arXiv:hep-ph/0606154.

[8] E. Aliu et al. [K2K Collaboration], Phys. Rev. Lett. 94, 081802 (2005) arXiv:hep-ex/0411038;

M. H. Ahn et al., arXiv:hep-ex/0606032.

[9] D.G. Michael et al. [MINOS Collaboration], arXiv:hep-ex/0607088.

[10] T. Futagami et al. [Super-Kamiokande Collaboration], Phys. Rev. Lett. 82, 5194 (1999) arXiv:astro-ph/9901139.

[11] See e.g., V. Barger, D. Marfatia and K. Whisnant, Phys. Lett. B 617, 78 (2005) arXiv:hep-ph/0501247.

[12] Q. R. Ahmad et al. [SNO Collaboration], Phys. Rev. Lett. 89, 011301 (2002) arXiv:nucl-ex/0204008]; S. N. Ahmed et al. [SNO Collaboration], Phys. Rev. Lett. 92, 181301 (2004) arXiv:nucl-ex/0309004]; B. Aharmim et al. [SNO Collaboration], Phys. Rev. C 72, 055502 (2005) arXiv:nucl-ex/0502021.

[13] B. Aharmim et al. [SNO Collaboration], Phys. Rev. D 72, 052010 (2005) arXiv:hep-ex/0507079.

[14] T. Araki et al. [KamLAND Collaboration], Phys. Rev. Lett. 94, 081801 (2005) arXiv:hep-ex/0406035. 